7th International Workshop on Astronomy and

Relativistic Astrophysics (IWARA 2016)

International Journal of Modern Physics: Conference Series

Vol. 45 (2017) 1760012 (4 pages)

(C) The Author(s)

DOI: $10.1142 / \mathrm{S} 2010194517600126$

\title{
Mimetic Dark Matter in Pseudo-Complex General Relativity
}

\author{
Guilherme Lorenzatto Volkmer and Dimiter Hadjimichef \\ Instituto de Fisica, Universidade Federal do Rio Grande do Sul (UFRGS) \\ Av. Bento Gonçalves, 9500, Porto Alegre, Rio Grande do Sul, 91501-970, Brazil \\ volkmer.guilherme@gmail.com,dimiter.hadjimichef@ufrgs.br
}

Published 15 August 2017

\begin{abstract}
We investigate the dark matter problem in the context of Pseudo-complex General Relativity. A form of gravitational dark matter has recently been studied, the mimetic dark matter, which is a scalar tensor extension for gravity where the conformal degree of freedom is isolated in a covariant way. For such, we perform a combination of both approaches to reveal non trivial results even in the absence of matter. Solutions for different scenarios and possible interpretations are presented.
\end{abstract}

Keywords: Dark Matter, Modified Theories of Gravity.

PACS numbers: 95.35. $+\mathrm{d}, 04.50 . \mathrm{Kd}$

\section{Introduction}

General Relativity predictions are in impressive agreement with experiments whose characteristic length scale ranges from microns $(\mu \mathrm{m})$ to about an astronomical unit (UA). ${ }^{1}$ On the other hand General relativity can only fit combined galactic, extragalactic and cosmological data if we assume a non vanishing cosmological constant and about six times more Dark Matter (matter which we have so far detected only through its gravitational interaction) than visible matter. ${ }^{1}$ Maybe the simplest way of pursuing a gravitational description that takes the dark matter problem in account is to promote General relativity to a scalar-tensor theory. It's worth to mention that theoretical physics provides a few candidates for the scalar field. ${ }^{2}$

\section{Mimetic Dark Matter}

Originally mimetic dark matter was introduced by rewriting the physical metric in terms of a scalar field and a auxiliary metric ${ }^{3}$

$$
g_{\mu \nu}=\left(\tilde{g}^{\alpha \beta} \partial_{\alpha} \varphi \partial_{\beta} \varphi\right) \tilde{g}_{\mu \nu} \equiv P \tilde{g}_{\mu \nu} .
$$

This is an Open Access article published by World Scientific Publishing Company. It is distributed under the terms of the Creative Commons Attribution 4.0 (CC-BY) License. Further distribution of this work is permitted, provided the original work is properly cited. 


\section{G. L. Volkmer \& D. Hadjimichef}

The metric (and therefore the resulting action of the theory) is invariant with respect of the conformal transformations of the auxiliary metric. With this modification the Einstein-Hilbert action becomes

$$
S=-\frac{1}{2} \int d^{4} x \sqrt{-g\left(\tilde{g}_{\mu \nu}, \varphi\right)}\left[R\left(g\left(\tilde{g}_{\mu \nu}, \varphi\right)\right)+\mathcal{L}_{m}\right] .
$$

Where $\mathcal{L}_{m}$ is the contribution due to ordinary matter and $R$ is the Ricci scalar. The subsequent equations of motion are given by:

$$
\begin{aligned}
G^{\mu \nu}-T^{\mu \nu}-(G-T) g^{\kappa \mu} g^{\lambda \nu} \partial_{\kappa} \varphi \partial_{\lambda} \varphi & =0, \\
\nabla_{\kappa}\left[(G-T) \partial^{\kappa} \varphi\right] & =0 .
\end{aligned}
$$

Aiming an interpretation of this extra degree of freedom we rewrite the first equation of motion:

$$
G^{\mu \nu}=T^{\mu \nu}+\tilde{T}^{\mu \nu} ; \quad \tilde{T}^{\mu \nu} \equiv(G-T) g^{\kappa \mu} g^{\lambda \nu} \partial_{\kappa} \varphi \partial_{\lambda} \varphi .
$$

Comparing $\tilde{T}^{\mu \nu}$ with the expression of the energy-momentum tensor of a perfect fluid $T^{\mu \nu}=(\epsilon+p) u^{\mu} u^{\nu}-p g^{\mu \nu}$ we can make the following association (with $p=0$ )

$$
\epsilon=G-T ; \quad u^{\mu}=g^{\mu \alpha} \partial_{\alpha} \varphi .
$$

Thus the mimetic dark matter extra degree of freedom behaves like a perfect fluid without pressure just like dark matter is described for large scales and sufficiently early times. ${ }^{4}$ Additionally it's worth to mention that the mimetic approach is different from other scalar-tensor works such as disformal gravity where the scalar field is dynamical. Here the scalar field is equivalent to the scaling factor up to an integrating constant. ${ }^{3}$

\section{Pseudo-Complex General Relativity}

Gauss observed that the generalization of real and complex numbers cannot be done without losing some peculiarities of algebraic operations. In particular, one must either renounce the commutative property of the product or acknowledge that the product between some non-zero numbers is zero. ${ }^{5}$ The pseudo-complex numbers fit the second case and are defined by ${ }^{5,6}$

$$
\left\{X=x+I y ; x, y \in \mathbb{R}, I \notin \mathbb{R} ; I^{2}=1\right\} .
$$

It's useful to work in a idempotent basis

$$
\sigma_{ \pm}=\frac{1}{2}(1 \pm I) ; \quad \sigma_{ \pm}^{2}=1, \quad \sigma_{+} \sigma_{-}=0
$$

The pseudo-complex variables in the idempotent basis are expressed in the form $X=$ $X_{+} \sigma_{+}+X_{-} \sigma_{-}$and a pseudo-holomorphic function (the analogous of holomorphic functions in the complex case) as $f(X)=f\left(X_{+}\right) \sigma_{+}+f\left(X_{-}\right) \sigma_{-}$. An important aspect of the pseudo-complex numbers are the "zero divisors", numbers that have only a component in the $\sigma_{+}$or $\sigma_{-}$sector. In the algebra of pseudo-complex numbers 
the zero divisors allow non-trivial solutions to the equation $X \cdot Y=0$. The metric tensor (assumed to be symmetric) can be written as:

$$
g_{\mu \nu}=g_{\mu \nu}^{+}\left(X^{+}\right) \sigma_{+}+g_{\mu \nu}^{-}\left(X^{-}\right) \sigma_{-} .
$$

And the pseudo-complex relations for other physical quantities can be found in the same fashion. If the variation of the action is set to zero the two independent components can be treated as two different theories and nothing new is obtained. For this formalism produce new results the variational principle has to be modified such that ${ }^{6}$

$$
\delta S=\delta S_{+} \sigma_{+}+\delta S_{-} \sigma_{-} \in \text { zero divisor } .
$$

The modified variational procedure gives an additional contribution to the Einstein field equations. It's an energy-momentum tensor with repulsive energy. Thus the modified Einstein equations are ${ }^{6}$

$$
G_{\mu \nu}=\Theta_{\mu \nu} \sigma_{-}, \quad \Theta_{\mu \nu}=\lambda\left[u_{\mu} u_{\nu}+\dot{y}_{\mu} \dot{y}_{\nu}-u_{\mu} \dot{y}_{\nu}-u_{\nu} \dot{y}_{\mu}\right] .
$$

Where $\lambda$ is a Lagrange multiplier. A mapping is provided to associate this extra tensor as a fluid ${ }^{6}$

$$
\left(T^{\text {fluid }}\right)_{\mu \nu}=\left(\epsilon_{\Lambda}+p_{\Lambda}\right) u_{\mu} u_{\nu}+p g_{\mu \nu}
$$

\section{Pseudo-Complex Mimetic Dark Matter}

The idea is to implement the mimetic metric in the pseudo-complex formalism

$$
g_{\mu \nu}=g_{\mu \nu}^{+}\left(X_{+}\right) \sigma_{+}+g_{\mu \nu}^{-}\left(X_{-}\right) \sigma_{-}=P^{+} \tilde{g}_{\mu \nu}^{+}\left(X_{+}\right) \sigma_{+}+P^{-} \tilde{g}_{\mu \nu}^{-}\left(X_{-}\right) \sigma_{-} .
$$

Applying the modified variational principle to this metric we see that the $\sigma_{+}$sector solution is the same of the original mimetic model but in the $\sigma_{-}$sector we have to consider the $\Theta_{\mu \nu} \sigma_{-}$contribution. The equations of motion acquire the form:

$$
\begin{aligned}
G^{\mu \nu}-T^{\mu \nu}-\Theta^{\mu \nu}-(G-T-\Theta) g^{\kappa \mu} g^{\lambda \nu} \partial_{\kappa} \varphi \partial_{\lambda} \varphi & =0, \\
\nabla_{\kappa}\left[(G-T-\Theta) \partial^{\kappa} \varphi\right] & =0 .
\end{aligned}
$$

Observe that in order to get the same functional form in both sectors we considered $\Theta_{\mu \nu}=\Theta_{\mu \nu} \sigma_{-}+\Theta_{\mu \nu} \sigma_{+}$and obviously all the componentes of $\Theta_{\mu \nu} \sigma_{+}$vanish. Now we have to furnish an interpretation for the extra degree of freedom including $\Theta_{\mu \nu}$. For this consider the energy-momentum tensor for a fluid with bulk viscosity ${ }^{7}$

$$
\Sigma^{\mu \nu}=\epsilon u^{\mu} u^{\nu}+p_{k} h^{\mu \nu}-\xi \nabla_{\gamma} u^{\gamma} h^{\mu \nu} .
$$

Where $h^{\mu \nu}=\left(u^{\mu} u^{\nu}+g^{\mu \nu}\right)$ and $\xi$ is the coefficient of bulk viscosity. With $p_{k}=0$ we can make another association, namely:

$$
\epsilon=G-T-\Theta ; \quad u^{\mu}=g^{\mu \alpha} \partial_{\alpha} \varphi ; \quad \Theta^{\mu \nu}=-\xi h^{\mu \nu} \nabla_{\gamma} u^{\gamma} .
$$

We take advantage from the repulsive character of $\Theta^{\mu \nu}$ to associate this contribution with bulk viscosity that is known to be able to induce negative pressure. In 
fact the Lagrange multiplier in (11) gives great liberty for the mapping to a fluid. Hence in the pseudo-complex extension of the mimetic model we can mimic viscous dark matter. Perhaps in this version of the model it's easier to treat the several inconsistencies that remains in the $\Lambda$ CDM like the "core-cusp" problem where the same simulations produce halo density profiles more cuspy than those measured in the center of dwarf galaxies. The inconsistencies imply that pressureless or cold dark matter (which the original model "mimics") produces an excess of structure and clustering compared to what we observe. ${ }^{8}$

\section{Conclusions}

The extra degree of freedom of the mimetic model solely yields an energy-momentum tensor that is related with a pressureless perfect fluid. A pseudo-complex approach for the mimetic model reveal the possibility of an association with viscous dark matter (vCDM), which is in agreement with cosmological observations. ${ }^{7}$ In addition, bulk viscosity has another important feature for cosmology: it can generate an accelerated expansion era without invoking dark energy. ${ }^{8}$ There are recent works that treated viscous dark matter in the domain of General relativity extesions. In Ref. ${ }^{9}$ is considered the interaction between a generalized Chaplygin gas with $f(R, T)$ gravity.

\section{References}

1. E. Papantonopoulos (ed.), Modifications of Einstein's Theory of Gravity at Large Distances, 1st edn. (Springer, New York, 2015).

2. Y. Fujii and K. Maeda, The Scalar-Tensor Theory of Gravitation, 1st edn. (Springer, New York, 2015).

3. A. H. Chamseddine and V. Mukhanov, J. High. Energy Phys. 11, 135 (2013).

4. F. Capela and S. Ramazanov, J. Cosmology. Astroparticle Phys. 1504, 051 (2015).

5. F. Catoni, D. Boccaletti, R. Cannata, V. Catoni, E. Nichelatti, and P. Zampetti, The Mathematics of Minkowski Space-Time: With an Introduction to Commutative Hypercomplex Numbers, 1st edn. (Birkhäuser Basel, Switzerland, 2008).

6. P. O. Hess, M. Schfer, and W. Greiner, Pseudo-Complex General Relativity, 1st edn. (Springer International Publishing, Switzerland, 2016).

7. H. Velten, Viscous Cold Dark Matter in Agreement with Observations, in Proc. 49th Winter School of Theoretical Physics, Cosmology and Non-Equilibrium Statistical Mechanics, (Ladek-Zdrój, Poland, 2014), p. 1460013.

8. G. Acquaviva, A. John, and A. Pnin, Phys. Rev. D94, 043517 (2016).

9. E. H. Baffou, I. G. Salako, and M. J. S. Houndjo, Viscous Generalized Chaplygin Gas Interacting with $f(R, T)$ Gravity, https://arxi v.org/abs/1606.05265. 